The authors conclude that patients with gout have an increased risk of death by all causes, and in particular by CVD-related death. These findings indicate that cardiovascular risk factors should be aggressively managed in patients with gout.

Original article Choi HK and Curhan G (2007)

Independent impact of gout on mortality and risk for coronary heart disease. Circulation 116: 894-900

\section{HAQ and PSHAQ scores and the prediction of disease activity in patients with RA}

The health assessment questionnaire (HAQ) is commonly used to assess the functional disability of patients with rheumatoid arthritis (RA), whereas the psychological HAQ (PSHAQ) has been developed to evaluate psychological distress in these patients. Verstappen et al. evaluated the association between clinical, demographic, functional and psychological factors and the HAQ and PSHAQ scores in a group of 112 patients with RA; the authors also assessed whether any of the factors evaluated at diagnosis were predictive of $\mathrm{HAQ}$ or PSHAQ score later on.

At diagnosis, clinical variables (erythrocyte sedimentation rate, Thompson joint score, visual analog score [VAS] for pain and for general well-being, and morning stiffness) and domains of psychological distress including anxiety were assessed; patients also completed the Dutch Health Assessment Questionnaire (VDF), a derivation of the original $\mathrm{HAQ}$, at this time. The same clinical variables were assessed at follow-up (7.1 \pm 3.4 years) at which time both $\mathrm{HAQ}$ and PSHAQ scores were obtained.

$\mathrm{HAQ}$ score was associated with all clinical variables at diagnosis and at follow-up. By contrast, PSHAQ score at follow-up was associated only with subjective patient-related clinical variables (VAS pain, VAS general wellbeing, and morning stiffness). In multivariate regression analyses, only functional disability (VDF score) at diagnosis was predictive of $\mathrm{HAQ}$ score at follow-up. Anxiety, but no clinical variable, assessed at diagnosis was predictive of PSHAQ score at follow-up.
The authors conclude that, in contrast to PSHAQ, HAQ score is a good indicator of disease activity at diagnosis and 7 years later in patients with RA.

Original article Verstappen SMM et al. (2007) Functional health assessment questionnaire (HAQ) and psychological HAQ are associated with and predicted by different factors in rheumatoid arthritis. J Rheumatol 34: 1837-1840

\section{ANS function is impaired in patients with Sjögren's syndrome}

Exocrine gland dysfunction in patients with Sjögren's syndrome does not always correlate with the extent of gland destruction; it has therefore been suggested that disturbances in other mechanisms, in particular the autonomic nervous system (ANS) that controls the exocrine glands, might be involved. A study by Mandl et al. has found evidence of sympathetic and parasympathetic neuropathy in patients with primary Sjögren's syndrome (pSS).

The study population consisted of 46 patients diagnosed with pSS according to the American-European Consensus Criteria. ANS function was tested by a deep breathing test, an orthostatic heart rate and blood pressure test, and a finger skin blood flow test. Compared with healthy controls, patients with pSS had significantly impaired sympathetic and parasympathetic nervous function. Surprisingly, however, when the exocrine function of patients with pSS was tested (by the unstimulated whole sialometry test, Schirmer-I test, and van Bijsterveld score), no correlation was found between ANS and exocrine function. The authors speculate that a lack of variability in exocrine function in the study population-most of whom had maximal exocrine dysfunction-might explain this finding. In addition, the present study measured cardiovascular ANS function; the authors suggest that tests specifically of exocrine ANS function might help to establish the relationship between exocrine and ANS function in patients with pSS.

Original article Mandl T et al. (2007) Autonomic and orthostatic dysfunction in primary Sjögren's syndrome. J Rheumatol 34: 1869-1874 